\title{
Article \\ Polycystic Ovary Syndrome Susceptibility Loci Inform Disease Etiological Heterogeneity
}

\author{
Yanfei Zhang ${ }^{1, *}$, Vani C. Movva ${ }^{2}$, Marc S. Williams ${ }^{1}$ and Ming Ta Michael Lee ${ }^{1}$ \\ 1 Genomic Medicine Institute, Geisinger, Danville, PA 17822, USA; mswilliams1@geisinger.edu (M.S.W.); \\ mlee2@geisinger.edu (M.T.M.L.) \\ 2 Department of Obstetrics and Gynecology, Geisinger Medical Center, Danville, PA 17822, USA; \\ vmovva@geisinger.edu \\ * Correspondence: zhyanfei123@gmail.com
}

check for updates

Citation: Zhang, Y.; Movva, V.C.; Williams, M.S.; Lee, M.T.M. Polycystic Ovary Syndrome Susceptibility Loci Inform Disease Etiological Heterogeneity. J. Clin. Med. 2021, 10, 2688. https://doi.org/10.3390/ jcm10122688

Academic Editor: Johannes Ott

Received: 15 May 2021

Accepted: 15 June 2021

Published: 18 June 2021

Publisher's Note: MDPI stays neutral with regard to jurisdictional claims in published maps and institutional affiliations.

Copyright: (c) 2021 by the authors. Licensee MDPI, Basel, Switzerland. This article is an open access article distributed under the terms and conditions of the Creative Commons Attribution (CC BY) license (https:// creativecommons.org/licenses/by/ $4.0 /)$.

\begin{abstract}
Polycystic ovary syndrome (PCOS) is a complex disorder with heterogenous phenotypes and unclear etiology. A recent phenotypic clustering study identified metabolic and reproductive subtypes of PCOS. We hypothesize that the heterogeneity of PCOS manifestations reflects different mechanistic pathways and can be identified using a genetic approach. We applied k-means clustering to categorize the genome-wide significant PCOS variants into clusters based on their associations with selected quantitative traits that likely reflect PCOS etiological pathways. We evaluated the association of each cluster with PCOS-related traits and disease outcomes. We then applied Mendelian randomization to estimate the causal effects between the traits and PCOS. Three categories of variants were identified: adiposity, insulin resistant, and reproductive. Significant associations were observed for variants in the adiposity cluster with body mass index (BMI), waist circumference and breast cancer, and variants in the insulin-resistant cluster with fasting insulin, glucose values, and homeostatic model assessment of insulin resistance (HOMA-IR). Sex hormone binding globulin (SHBG) has strong association with all three clusters. Mendelian randomization suggested a causal role of BMI and SHBG on PCOS. No causal associations were observed for PCOS on disease outcomes.
\end{abstract}

Keywords: polycystic ovary syndrome; clustering; genetic heterogeneity; adiposity; insulin resistance; Mendelian randomization; causality; sex hormone binding globulin

\section{Introduction}

Polycystic ovary syndrome (PCOS) is a complex disorder affecting approximately $15 \%$ of women of reproductive age [1]. PCOS includes highly heterogeneous phenotypic manifestations characterized by a variety of reproductive and metabolic abnormalities, including ovulatory dysfunction, hyperandrogenism, hirsutism, obesity, and insulin resistance [1]. The commonly used National Institutes of Health (NIH) [2] and Rotterdam [3,4] diagnostic criteria for PCOS are designed to account for the diverse phenotypic presentations but do not provide mechanistic insights [5]. The etiology or etiologies of PCOS are still unclear.

To obtain insight into the etiology and deconstruct the heterogeneity of PCOS, a recent study performed clustering analysis using body mass index (BMI) and seven biochemical biomarkers in a PCOS cohort and identified two distinct phenotypic clusters: a "reproductive" subtype characterized by high luteinizing hormone (LH) and sex hormone binding globulin (SHBG) levels with low BMI and insulin levels, and a "metabolic" subtype characterized by high BMI, glucose, and insulin levels with low SHBG and LH levels [5]. It is important to note that biochemical markers change with many factors, such as aging, certain metabolic traits, such as obesity and type 2 diabetes, and use of insulin and contraceptive pills. Given the observational nature of cross-sectional studies, it is also unclear whether these biomarkers are causal or consequential to the disease. Unlike biomarkers, germline DNA remains constant regardless of external factors and age. Thus, genetic variants are often used as variables to explore causality. 
PCOS is highly heritable with an estimated heritability of $38-71 \%$ as noted in the twin study [6]. Recent large-scale genome-wide association studies (GWAS) brought significant progress in identification of PCOS susceptibility loci [7-12]. Thirty-seven variants with genome-wide significance have been identified so far by GWAS in European and East Asian populations, offering insights into causal biological pathways for PCOS. With the public access to GWAS dataset of many traits and disease outcomes, it is now possible to elucidate disease mechanisms using variant clustering techniques assuming that genetic variants that act along a shared pathway will have similar directional effect on a trait [13]. Such a strategy was previously applied to deconstruct the mechanistic heterogeneity of type 2 diabetes mellitus (T2DM) [13-15].

In this study, we hypothesize that the heterogeneity of PCOS manifestations reflects different mechanistic pathways and can be identified using a genetic approach. We performed clustering analysis on the association of PCOS susceptibility loci with various traits that are related to PCOS. We then used genetic risk scoring to evaluate the effect of each cluster. We further performed Mendelian randomization to estimate the causal effect of the traits on PCOS and PCOS on disease outcomes.

\section{Materials and Methods}

\subsection{Selection of PCOS-Associated Genetic Variants, Traits and Disease Outcomes}

We compiled a list of 37 genome-wide significant variants associated with PCOS $\left(p<5 \times 10^{-8}\right)$ from previously published GWAS (Supplementary Table S1). Only variants that are not in linkage disequilibrium $\left(\mathrm{LD} \mathrm{R}^{2}<0.5\right)$ were included. Four variants were excluded later as they were not included in the summary statistics of most of the traits or disease outcomes, and no proxy single nucleotide variants (SNVs) could be identified for them. A total of 26 variants were included in the final analysis. The single nucleotide variants (SNVs) rs10993397 (C9orf3) and rs8043701 (TOX3) were replaced by their proxy SNVs rs7865239 and rs11075468 (Supplementary Table S1).

We selected four groups of traits that are likely to inform PCOS etiologies: (1) adiposity traits: female body mass index (BMI, general adiposity), female waist circumstances (WC) and female waist hip ratio (WHR, central adiposity) [16,17]; (2) hormonal traits: sex hormone binding globulin (SHBG), luteinizing hormone (LH) [18]; (3) insulin-resistant traits: fasting insulin (FI), fasting glucose (FG), homeostatic model assessment of insulin resistance (HOMA-IR) [19,20]; (4) lipids: high-density lipoprotein (HDL), low-density lipoprotein (LDL), total cholesterol (TC) and triglycerides (TG) [21]. Disease outcomes include T2DM [22], coronary artery disease (CAD) [23], and breast cancer [24]. Traits and outcomes such as follicle stimulating hormone (FSH), testosterone, dehydroepiandrosterone sulfate (DEHAS), and other female reproductive organ cancers, such as endometrial and ovarian cancer, although planned, could not be included as the GWAS datasets were unavailable or included very few PCOS susceptibility loci and proxy SNVs. The TwoSampleMR package was developed to ease Mendelian randomization analysis [25]. It connects to the IEU open GWAS database, making it convenient to extract and harmonize data. We employed the TwoSampleMR package to retrieve, read in, and harmonize the association summary statistics of PCOS variants with these traits and outcomes. All the datasets used in this study are publicly available and the resources are provided in Supplementary Table S2.

\subsection{Clustering Analysis}

First, we calculated the z-score ( $z$-score $=\beta /$ se) from the summary statistics of the 26 PCOS variants from the GWAS of the four groups of quantitative traits (Supplementary Table S3). All effects were aligned to the PCOS risk-increasing alleles. We then applied $k$-means clustering on the association $\mathrm{z}$-scores where variants are clustered together based on the similar associations with the traits. This method is widely applied to quantitative data and was previously used by our team to identify subgroups of patients with different responses to phenylephrine [26]. As k-means clustering requires defining the number of clusters in advance, we used the NbClust package to decide the best num- 
ber of clusters [27]. Supplementary Figure S1 shows that 8 indices suggest a three-cluster solution. Analyses were performed using R (version 3.6.3).

\subsection{Trait and Disease Associations with Each Cluster}

The association of the genetic risk scores of each cluster with each trait and disease outcome was performed by an inverse-variance fixed effect meta-analysis of the summary statistics of the variant-trait and variant-disease from GWAS described previously [13-15]. Association with five disease outcomes, which were not used in clustering analysis were also examined. $P$-value $<0.0024$ is considered significant with Bonferroni correction for 16 traits and 5 disease outcomes $(0.05 / 21)$.

\subsection{Mendelian Randomization Analysis}

Based on the association results of genetic risk score with traits and disease outcomes, we performed Mendelian randomization analysis to evaluate the causal role of SHBG, BMI, WC, WHR, and insulin resistance on PCOS. Instrumental variables for SHBG, BMI, WC, WHR were extracted from the curated dataset by the IEU open GWAS project (Supplementary Table S2). For insulin resistance, we used the 53 significant variants associated with an integrated insulin-resistant phenotype composed of FI, TG, and HDL [28]. We adopted the $\beta$ and SE from the study of Wang et al., who meta-analyzed the absolute value of the standardized $\beta$ coefficient for each of the 53 SNV associations with the individual components of the composite IR phenotype using a fixed-effect inverse-variance-weighted (IVW) method (Supplementary Table S4) [29]. When evaluating the causal roles of these traits on PCOS, we meta-analyzed the results from studies by Day et al. (without samples from 23andme) [11] and Zhang et al., [12] using METAL [30] to increase the GWAS sample size for PCOS. When evaluating the causal role of PCOS on disease outcomes, we used the 14 variants reported from the largest meta-analysis for PCOS as instrumental variables [11]. TwoSampleMR R package was used to perform MR using the inverse-variance-weighted (IVW) method for main analysis and MR-Egger and weighted median methods for sensitivity analyses to evaluate the robustness [25]. $P$-value $<0.05$ is considered significant.

\section{Results}

\subsection{Clustering Suggests Mechanistic Heterogeneity for PCOS Etiology}

Three variant clusters were identified by $k$-means clustering on association z-scores of 26 PCOS variants and 16 traits. These clusters were mainly distinguished by the BMI-related traits, insulin-resistant traits, and SHBG, as visualized in the PCA plot (Figure 1). Thus, we named them "adiposity", "insulin resistant" and "reproductive" clusters. Table 1 provides the association statistics of genetic risk score of each cluster and the traits. The adiposity cluster includes three variants in DENND1A and one variant in FSHR. BMI $\left(\beta=0.015, p=2.59 \times 10^{-7}\right)$ and WC $\left(\beta=0.017, p=1.67 \times 10^{-7}\right)$ are the most significantly associated traits. WC remains significant even with BMI adjustment $(\beta=0.01$, $p=0.001)$. WHR is only significant without BMI adjustment $(\beta=0.011, p=0.0008)$. Additionally, the adiposity cluster is negatively associated with SHBG $(\beta=-0.198$, $\left.p=7.34 \times 10^{-6}\right)$. The insulin-resistant cluster has 10 variants in nine genes including THADA (two variants), LHCGR, FSHB, FSHR, ERBB3, TOX3, GATA4, HMGA2, and KRR1. Fasting insulin is the most significant trait, both with and without BMI adjustment ( $\beta=0.004, p=2.12 \times 10^{-5} ; \beta=0.005, p=1.93 \times 10^{-5}$, respectively). HOMA-IR $(\beta=0.005$, $p=0.0013)$, fasting glucose $(\beta=0.004, p=0.0004)$ and total cholesterol $(\beta=-0.006$, $p=0.0009$ ) are only significant without BMI adjustment. The reproductive cluster includes 12 variants in 11 genes of ERBB4, RAB5B, IRF1, SOD2, YAP1, SUMO1B, ZBTB16, C9orf3 (2 variants), INSR, THADA, and TOX3. SHBG is the only significant trait associated with this cluster $\left(\beta=0.0052, p=1.70 \times 10^{-6}\right)$. 


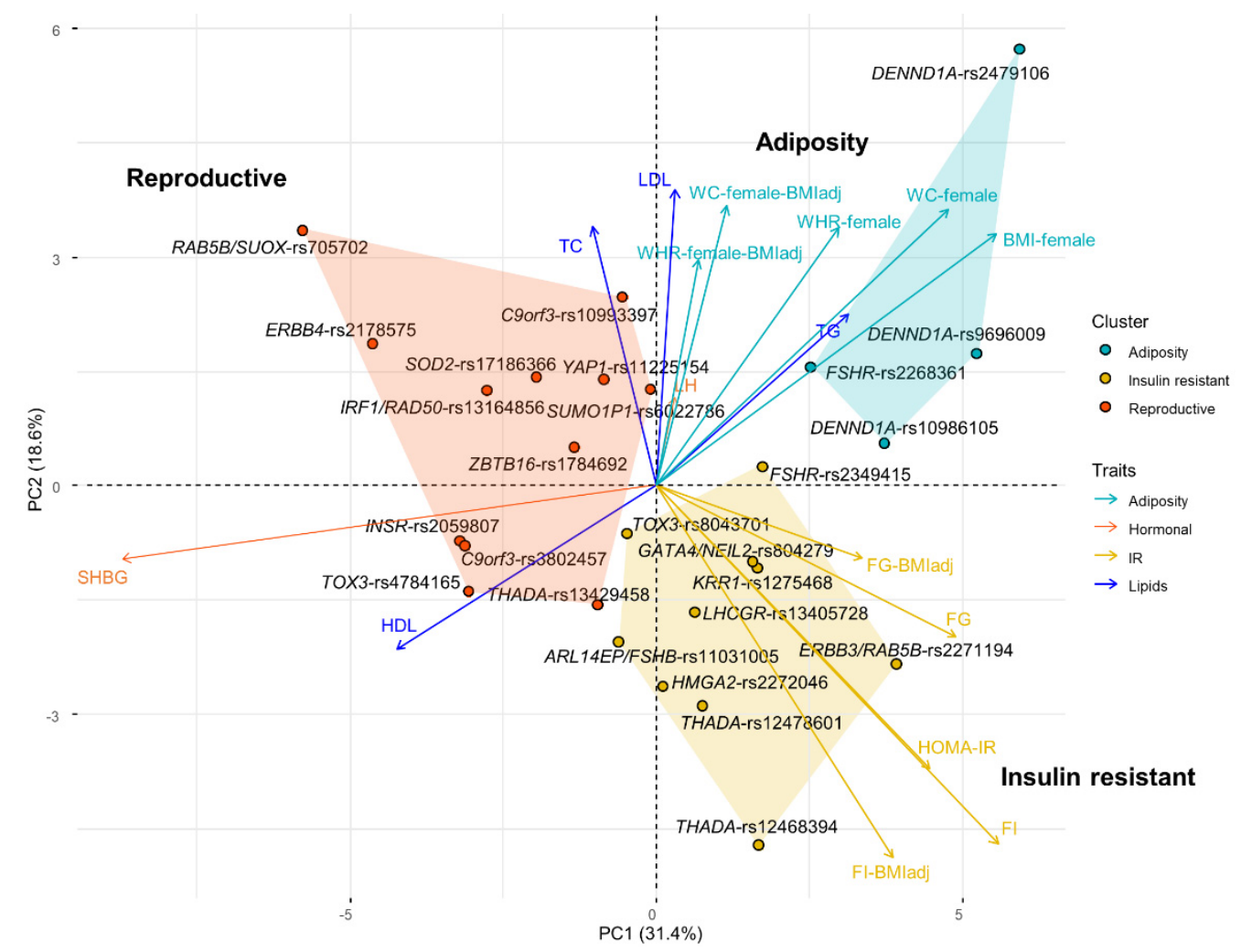

Figure 1. PCA plot of the variant-trait associations for PCOS variants. PCOS variants are plotted on the first 2 principal components (PCs) of the association Z-score and colored by the assigned clusters. The relative magnitude and direction of trait correlation with the PCs are shown with arrows. BMI, body mass index; WC: waist circumference; WHR: waist-hip ratio; PC, principal component; PCA, principal component analysis; FG: fasting glucose; FI: fasting insulin; SHBG, sex hormone binding globulin; LH, luteinizing hormone; HOMA-IR: homeostatic model assessment of insulin resistance; FI: fasting insulin; FG: fasting glucose; HDL: high-density lipoprotein; LDL: low-density lipoprotein; TC: total cholesterol; TG: triglycerides.

We also investigated the association of clusters with disease outcomes (Table 1). None of the three clusters are associated with CAD or T2DM. The adiposity cluster is significantly associated with breast cancer $(\beta=0.014, p=0.0008)$. The insulin-resistant and reproductive clusters are associated with breast cancer at nominal significance $(p<0.05)$.

\subsection{Mendelian Randomization Suggests a Causal Role of SHBG and BMI on PCOS}

The clustering analysis suggests that BMI, insulin resistance, and SHBG are involved in PCOS etiology. We applied MR to further estimate the causal roles of these factors on PCOS. The inverse-variance-weighted method suggests a causal effect of SHBG, BMI, WC, and insulin resistance (Table 2). In the sensitivity analysis using the weighted median and MR-Egger methods, only SHBG and BMI remained significant $(p<0.05)$. SHBG shows a mild protective effect (odds ratio $(\mathrm{OR})<1$ ) on PCOS with an OR of $0.988,95 \%$ confidence interval (CI) of 0.981 to 0.995 , per $1 \mathrm{nmol} / \mathrm{L}$ higher SHBG ( $p=6.694 \times 10^{-4}$, Table 2). BMI shows a moderate risk effect with an OR of $2.421,95 \%$ CI of 1.910 to 3.068 , per 1 standard deviation (SD), which is $4.77 \mathrm{~kg} / \mathrm{m}^{2}$ in the original GWAS cohort, and an increase in BMI $\left(p=2.611 \times 10^{-13}\right.$, Table 2$)$. Some epidemiologic studies have reported increased risk for T2DM, CAD and breast cancer in women with PCOS. Therefore, we evaluated the causal effect of PCOS on disease outcomes. We did not observe significant evidence to support a causal role for PCOS on T2DM or CAD. According to the IVW results, PCOS shows a causal effect on breast cancer $(\beta=0.0646, p=0.00195)$, especially the estrogen receptor $(\mathrm{ER})$ positive type $\left(\beta=0.0862, p=7.9 \times 10^{-3}\right)$. However, the MR-Egger results are no longer significant, suggesting pleiotropic effect of the instrumental variants. 
Table 1. The association of the genetic risk score of each cluster with traits and disease outcomes.

\begin{tabular}{|c|c|c|c|c|c|c|}
\hline & \multicolumn{2}{|c|}{ Adiposity } & \multicolumn{2}{|c|}{ Insulin Resistant } & \multicolumn{2}{|c|}{ Reproductive } \\
\hline & $\beta$ & $p$-Value & $\beta$ & $p$-Value & $\beta$ & $p$-Value \\
\hline SHBG & -0.198 & $7.377 \times 10^{-6}$ & -0.069 & 0.0052 & 0.111 & $1.70 \times 10^{-6}$ \\
\hline LH & 0.025 & 0.1462 & 0.002 & 0.8053 & -0.001 & 0.8898 \\
\hline FI & 0.004 & 0.0855 & 0.005 & $1.93 \mathrm{E}-05$ & -0.003 & 0.0101 \\
\hline FI-BMI adj. & 0.002 & 0.3702 & 0.004 & 2.12E-05 & -0.001 & 0.1558 \\
\hline FG & 0.004 & 0.0519 & 0.004 & 0.0004 & -0.002 & 0.0653 \\
\hline FG-BMI adj. & 0.003 & 0.1947 & 0.003 & 0.0138 & -0.001 & 0.3067 \\
\hline HOMA-IR & 0.003 & 0.2389 & 0.005 & 0.0013 & -0.003 & 0.0633 \\
\hline HDL & -0.008 & 0.0035 & 0 & 0.9734 & 0.003 & 0.0411 \\
\hline LDL & 0.003 & 0.335 & -0.004 & 0.0152 & -0.002 & 0.2336 \\
\hline TG & 0.001 & 0.6019 & -0.003 & 0.0499 & -0.004 & 0.0207 \\
\hline $\mathrm{TC}$ & -0.001 & 0.7885 & -0.006 & 0.0009 & -0.001 & 0.5062 \\
\hline BMI & 0.015 & $2.59 \times 10^{-7}$ & 0 & 0.9586 & -0.001 & 0.4855 \\
\hline WC & 0.017 & $1.67 \times 10^{-7}$ & 0 & 0.892 & 0.001 & 0.7129 \\
\hline WC-BMI adj. & 0.01 & 0.001 & 0 & 0.8755 & 0.004 & 0.0264 \\
\hline WHR & 0.011 & 0.0008 & -0.002 & 0.3264 & 0 & 0.8949 \\
\hline WHR-BMI adj. & 0.006 & 0.0807 & -0.002 & 0.251 & 0.001 & 0.5162 \\
\hline CAD & -0.007 & 0.1482 & 0 & 0.8988 & 0 & 0.8444 \\
\hline T2DM & 0.009 & 0.0726 & -0.004 & 0.2279 & -0.005 & 0.0964 \\
\hline Breast cancer & 0.014 & 0.0008 & 0.007 & 0.0106 & 0.006 & 0.0192 \\
\hline ER positive & 0.012 & 0.0201 & 0.008 & 0.0146 & 0.005 & 0.0864 \\
\hline ER negative & 0.016 & 0.0423 & 0.002 & 0.6789 & 0.003 & 0.4304 \\
\hline
\end{tabular}

Adj.: adjusted; WC: waist circumference; WHR: waist-hip ratio; BC: breast cancer; ER: estrogen receptor; T2DM: type 2 diabetes mellitus; CAD: coronary artery disease; SHBG: sex hormone binding globulin; BMI: body mass index; LH: luteinizing hormone; HOMA-IR: homeostatic model assessment of insulin resistance; FI: fasting insulin; FG: fasting glucose; HDL: high-density lipoprotein; LDL; low-density lipoprotein; TG: triglyceride; TC: total cholesterol.

Table 2. Result of Mendelian randomization of traits on PCOS and PCOS on disease outcomes.

\begin{tabular}{ccccccc}
\hline Trait & Method & nSNV & $\boldsymbol{\beta}$ & SE & OR (95\%CI) & $p$-Value \\
\hline SHBG & MR Egger & 171 & -0.0140 & 0.0067 & $0.986[0.973,0.999]$ & $3.926 \times 10^{-2}$ \\
SHBG & Weighted median & 171 & -0.0162 & 0.0052 & $0.984[0.974,0.994]$ & $1.785 \times 10^{-3}$ \\
SHBG & IVW & 171 & -0.0120 & 0.0035 & $0.988[0.981,0.995]$ & $6.694 \times 10^{-4}$ \\
BMI-female & MR Egger & 35 & 1.2206 & 0.3224 & $3.389[1.802,6.376]$ & $6.157 \times 10^{-4}$ \\
BMI-female & Weighted median & 35 & 0.9210 & 0.1887 & $2.512[1.735,3.636]$ & $1.056 \times 10^{-6}$ \\
BMI-female & IVW & 35 & 0.8842 & 0.1209 & $2.421[1.910,3.068]$ & $2.611 \times 10^{-13}$ \\
Insulin resistance & MR Egger & 51 & 0.5460 & 0.4531 & $1.726[0.710,4.196]$ & $2.340 \times 10^{-1}$ \\
Insulin resistance & Weighted median & 51 & 0.1011 & 0.2687 & $1.106[0.653,1.873]$ & $7.067 \times 10^{-1}$ \\
Insulin resistance & IVW & 51 & 0.5267 & 0.2220 & $1.693[1.096,2.616]$ & $1.768 \times 10^{-2}$ \\
WC-female & MR Egger & 18 & 0.9313 & 0.7627 & $2.538[0.569,11.316]$ & $2.398 \times 10^{-1}$ \\
WC-female & Weighted median & 18 & 0.6591 & 0.2646 & $1.933[1.151,3.247]$ & $1.276 \times 10^{-2}$ \\
WC-female & IVW & 18 & 0.5738 & 0.2112 & $1.775[1.173,2.685]$ & $6.596 \times 10^{-3}$ \\
BMI adj. WC-female & MR Egger & 24 & 0.6659 & 0.7861 & $1.946[0.417,9.085]$ & $4.061 \times 10^{-1}$ \\
BMI adj. WC-female & Weighted median & 24 & 0.2865 & 0.2213 & $1.332[0.863,2.055]$ & $1.955 \times 10^{-1}$ \\
BMI adj. WC-female & IVW & 24 & 0.3255 & 0.1774 & $1.385[0.978,1.961]$ & $6.650 \times 10^{-2}$ \\
WHR-female & MR Egger & 20 & -0.7122 & 1.1173 & $0.491[0.055,4.383]$ & $5.319 \times 10^{-1}$ \\
WHR-female & Weighted median & 20 & 0.2172 & 0.2383 & $1.243[0.779,1.982]$ & $3.619 \times 10^{-1}$ \\
WHR-female & IVW & 20 & 0.3912 & 0.2220 & $1.479[0.957,2.285]$ & $7.806 \times 10^{-2}$ \\
BMI adj. WHR-female & MR Egger & 32 & 0.0927 & 0.5285 & $1.097[0.389,3.091]$ & $8.620 \times 10^{-1}$ \\
BMI adj. WHR-female & Weighted median & 32 & 0.1742 & 0.1743 & $1.190[0.846,1.675]$ & $3.175 \times 10^{-1}$ \\
BMI adj. WHR-female & IVW & 32 & 0.2089 & 0.1387 & $1.232[0.939,1.617]$ & $1.322 \times 10^{-1}$ \\
\hline
\end{tabular}


Table 2. Cont.

\begin{tabular}{ccccccc}
\hline Outcome & Method & nSNP & $\boldsymbol{\beta}$ & SE & OR (95\%CI) & $p$-Value \\
\hline T2DM & MR Egger & 8 & 0.0244 & 0.1823 & $1.025[0.717,1.465]$ & $8.979 \times 10^{-1}$ \\
T2DM & Weighted median & 8 & -0.0285 & 0.0355 & $0.972[0.907,1.042]$ & $4.208 \times 10^{-1}$ \\
T2DM & IVW & 8 & -0.0325 & 0.0384 & $0.968[0.898,1.044]$ & $3.962 \times 10^{-1}$ \\
CAD & MR Egger & 10 & -0.0226 & 0.1086 & $0.978[0.790,1.210]$ & $8.403 \times 10^{-1}$ \\
CAD & Weighted median & 10 & -0.0474 & 0.0283 & $0.954[0.902,1.008]$ & $9.473 \times 10^{-2}$ \\
CAD & IVW & 10 & -0.0349 & 0.0228 & $0.966[0.923,1.010]$ & $1.258 \times 10^{-1}$ \\
BC & MR Egger & 9 & 0.0441 & 0.1278 & $1.045[0.814,1.343]$ & $7.403 \times 10^{-1}$ \\
BC & Weighted median & 9 & 0.0727 & 0.0268 & $1.075[1.020,1.133]$ & $6.697 \times 10^{-3}$ \\
BC & IVW & 9 & 0.0646 & 0.0277 & $1.067[1.010,1.126]$ & $1.950 \times 10^{-2}$ \\
ER + BC & MR Egger & 9 & 0.0859 & 0.1501 & $1.090[0.812,1.462]$ & $5.850 \times 10^{-1}$ \\
ER + BC & Weighted median & 9 & 0.0981 & 0.0341 & $1.103[1.032,1.179]$ & $4.015 \times 10^{-3}$ \\
ER + BC & IVW & 9 & 0.0862 & 0.0324 & $1.090[1.023,1.161]$ & $7.900 \times 10^{-3}$ \\
ER - BC & MR Egger & 9 & -0.0564 & 0.1630 & $0.945[0.687,1.301]$ & $7.394 \times 10^{-1}$ \\
ER - BC & Weighted median & 9 & 0.0168 & 0.0399 & $1.017[0.940,1.100]$ & $6.744 \times 10^{-1}$ \\
ER - BC & IVW & 9 & 0.0605 & 0.0368 & $1.062[0.988,1.142]$ & $1.001 \times 10^{-1}$ \\
\hline
\end{tabular}

Adj.: adjusted; IVW: inverse variance weighted; WC: waist circumference; WHR: waist-hip ratio; BC: breast cancer; ER: estrogen receptor; T2DM: type 2 diabetes mellitus; CAD: coronary artery disease; SHBG: sex hormone binding globulin; BMI: body mass index; SE: standard error; OR: odds ratio; CI: confidence interval.

\section{Discussion}

Common complex diseases consist of combinations of symptoms and phenotypes that may result from different mechanistic pathways. Several studies have identified subtypes of T2DM using biomarkers or phenotypes [31,32]. These subtypes indicate different mechanistic pathways of T2DM, which were later supported by deconstruction of T2DM susceptibility loci $[13,15]$. In this study, we performed a clustering analysis on PCOS susceptibility variants and identified three clusters of variants that associate with adiposity, insulin-resistant and hormonal traits, providing genetic evidence for the recently reported metabolic and reproductive subtypes of PCOS in a phenotypic clustering using BMI and seven serum biochemical markers [5].

Similar to the phenotypic clustering study where BMI, fasting insulin, and SHBG are the key features separating the metabolic and reproductive subtypes [5], the clusters of variants in our analysis were mainly differentiated by associations with SHBG, BMI, and fasting insulin. However, in the prior study, BMI and fasting insulin were clustered together as the metabolic subtype, and thus could not be separated by phenotypic clustering. In this analysis, the adiposity and insulin-resistant clusters were clearly separated by associations with BMI and fasting insulin, suggesting a higher resolution for the genetic variant clustering than the phenotypic clustering. In the phenotypic clustering, the metabolic subtype showed relatively higher BMI, fasting insulin, and lower SHBG, while the reproductive subtype showed higher SHBG but lower BMI and fasting insulin. The current study provides orthogonal evidence to phenotypic clustering as evidenced by the directionally concordant results where the adiposity and insulin-resistant clusters showed significant positive associations with BMI and fasting insulin but negative association with SHBG, while the reproductive cluster showed significant positive association with SHBG and negative association with fasting insulin $(p=0.01)$ and a consistent direction of association with BMI (not significant, $p=0.4855$ ). We also investigated the role of fat distribution patterns using WC and WHR. WC is significantly associated with the adiposity cluster even after BMI adjustment. However, the association with WHR became nonsignificant with BMI adjustment. These results suggest that general adiposity, but not central adiposity, is the main associated feature.

In the MR analysis, we confirmed the causal association of BMI on PCOS by the IVW and MR Egger methods, which are consistent with previous studies [9,33,34]. To investigate the causal effect of insulin resistance, we used the 53 variants associated with an integrated insulin-resistant phenotype that included three components-high levels of fasting insulin 
and TG, low levels of HDL [28]. Using the IVW method, we identified a potential causal relationship between insulin resistance and PCOS, although the weighted median and MR-Egger test suggested that the causal effect might be driven by genetic pleiotropy, for example, the potential pleotropic effect on BMI. However, accumulating evidence suggests insulin resistance as an important mechanism, and several insulin sensitizing drugs have been used to ameliorate PCOS symptoms and signs, including metformin, the first insulin sensitizing drug used in PCOS [35], and inositol isoforms, evidenced by several recent studies showing high safety profile and effectiveness [36,37].

We also explored the association of variant clusters with the disease outcomes. Epidemiologic studies showed increased risk of diabetes in patients with PCOS [38,39]. However, these studies often suffer from unknown confounding factors making it difficult to infer causality. In our analysis, we did not observe significant associations between variant clusters and T2DM. MR also did not identify a potential causal effect of PCOS on T2DM. Conflicting results were observed between PCOS and breast cancer [40]. To date, studies have not observed an association between PCOS and breast cancer risk [41]. In our study, we observed marginal associations of breast cancer with three variant clusters. The subsequent MR-Egger analysis suggested that the effect of PCOS on breast cancer is possibly mediated by other factors, such as BMI and estrogen.

Our study has limitations. First, we used k-means clustering, which is a "hardclustering" method where one variant is assigned to only one cluster. However, this does not reflect the reality most of the time as a single gene can be involved in multiple pathways (pleiotropy). In our analysis, we observed that different variants from the same genes are classified into different clusters, such as variants in THADA, FSHR, and TOX3. Second, our study is limited by the GWAS datasets available for PCOS-related traits, especially gonadotropin. Only SHBG and LH were included in our analysis. FSH, testosterone and DEHAS, which are also important hormonal traits for PCOS, were not analyzed as the corresponding GWAS dataset was either unavailable or had very few PCOS-associated variants. The SHBG protein level varied largely depending on factors including age, BMI, insulin resistance and liver diseases. However, the original GWAS of SHBG in our analysis by the Neale lab using UK biobank data was only adjusted for sex, but not for BMI or insulin resistance. Even though MR analysis suggested causal association of SHBG with PCOS, future SHBG GWAS with a larger sample size and proper adjustment will be required to definitively establish a causal association. There was no association with LH in the variant clustering as observed in the phenotypic clustering. FSH and LH vary significantly between individuals and changes rapidly in response to physiologic factors such as menstrual cycle, which cannot be easily accounted for in these types of analyses [42]. A GWAS of LH with small sample size may lack statistical power to capture the variabilities nature of LH levels in the general population. This may explain why variants in FSHR, FSHB, and LHCGR do not cluster together. Furthermore, GWAS of PCOS-related disease outcomes, such as hirsutism, endometrial and ovarian cancer, are not available or have a limited number of variants, thus we were unable to evaluate the associations of genetic risk score of each cluster with these outcomes. With increasing access to more large-scale GWAS with more granular features, future studies can apply a more sophisticated "soft-clustering" method on more non-disease quantitative traits that hopefully can overcome the limitations of this study.

\section{Conclusions}

Our study is the first to use a genetic approach to deconstruct PCOS etiological heterogeneity. Clustering of variants associated with PCOS has identified three likely etiologic pathways involving adiposity, insulin resistance and SHBG. Subsequent MR analysis suggests a causal role for BMI and SHBG and a suggestive causal effect of insulin resistance on PCOS. Studies such as this will accelerate the deep phenotyping of PCOS and could inform diagnostic criteria that currently do not distinguish the subtypes of PCOS. If 
successful, this could help to classify women with PCOS and improve treatment precision in future.

Supplementary Materials: The following are available online at https:/ / www.mdpi.com/article / 10.3390/jcm10122688/s1, Supplementary Figure S1: Using NbClust to identify the best number of clusters for k-means clustering, Supplementary Table S1: PCOS significant variants by GWAS, Supplementary Table S2: List of GWAS datasets of PCOS-related traits and disease outcomes, Supplementary Table S3: Z score for 26 PCOS SNV-quantitative trait associations, Supplementary Table S4: The 53 instrumental variables for composed insulin-resistant phenotype.

Author Contributions: Conceptualization, Y.Z.; data curation, Y.Z. and V.C.M.; formal analysis, Y.Z.; investigation, M.S.W. and M.T.M.L.; methodology, Y.Z.; validation, V.C.M., M.S.W. and M.T.M.L.; writing—original draft, Y.Z.; writing—review and editing, V.C.M., M.S.W. and M.T.M.L. All authors have read and agreed to the published version of the manuscript.

Funding: This research received no external funding.

Institutional Review Board Statement: Ethical review and approval were waived for this study due to only publicly available summary statistical datasets being used.

Informed Consent Statement: Not applicable.

Data Availability Statement: Data used in this study are publicly available. The dataset link or ID numbers are provided in the Supplementary Table S2. The generated data used for clustering analysis are provided in the Supplementary Table S3.

Acknowledgments: The authors express their gratitude to the IEU open GWAS project (https: / / gwas.mrcieu.ac.uk/; last accessed on 28 October 2020.) and the consortium and teams that shared the GWAS summary statistics.

Conflicts of Interest: The authors declare no conflict of interest.

\section{References}

1. Diamanti-Kandarakis, E.; Dunaif, A. Insulin resistance and the polycystic ovary syndrome revisited: An update on mechanisms and implications. Endocr. Rev. 2012, 33, 981-1030. [CrossRef]

2. Zawadzki, J.K.; Dunaif, A. Diagnostic Criteria for Polycystic Ovary Syndrome: Towards a Rational Approach; Dunaif, A.G.J., Haseltine, F.P., Merriam, G.R., Eds.; Blackwell Scientific Publications: Boston, MA, USA, 1992.

3. Rotterdam ESHRE; ASRM-Sponsored PCOS Consensus Workshop Group. Revised 2003 consensus on diagnostic criteria and long-term health risks related to polycystic ovary syndrome. Fertil. Steril. 2004, 81, 19-25. [CrossRef] [PubMed]

4. Rotterdam ESHRE; ASRM-Sponsored PCOS Consensus Workshop Group. Revised 2003 consensus on diagnostic criteria and long-term health risks related to polycystic ovary syndrome (PCOS). Hum. Reprod. 2004, 19, 41-47. [CrossRef]

5. Dapas, M.; Lin, F.T.J.; Nadkarni, G.N.; Sisk, R.; Legro, R.S.; Urbanek, M.; Hayes, M.G.; Dunaif, A. Distinct subtypes of polycystic ovary syndrome with novel genetic associations: An unsupervised, phenotypic clustering analysis. PLoS Med. 2020, 17, e1003132. [CrossRef]

6. Vink, J.M.; Sadrzadeh, S.; Lambalk, C.B.; Boomsma, D.I. Heritability of polycystic ovary syndrome in a Dutch twin-family study. J. Clin. Endocrinol. Metab. 2006, 91, 2100-2104. [CrossRef] [PubMed]

7. Chen, Z.J.; Zhao, H.; He, L.; Shi, Y.; Qin, Y.; Shi, Y.; Li, Z.; You, L.; Zhao, J.; Liu, J.; et al. Genome-wide association study identifies susceptibility loci for polycystic ovary syndrome on chromosome 2p16.3, 2 p21 and 9q33.3. Nat. Genet. 2011, 43, 55-59. [CrossRef]

8. Shi, Y.; Zhao, H.; Shi, Y.; Cao, Y.; Yang, D.; Li, Z.; Zhang, B.; Liang, X.; Li, T.; Chen, J.; et al. Genome-wide association study identifies eight new risk loci for polycystic ovary syndrome. Nat. Genet. 2012, 44, 1020-1025. [CrossRef]

9. Day, F.R.; Hinds, D.A.; Tung, J.Y.; Stolk, L.; Styrkarsdottir, U.; Saxena, R.; Bjonnes, A.; Broer, L.; Dunger, D.B.; Halldorsson, B.V.; et al. Causal mechanisms and balancing selection inferred from genetic associations with polycystic ovary syndrome. Nat. Commun. 2015, 6, 8464. [CrossRef]

10. Hayes, M.G.; Urbanek, M.; Ehrmann, D.A.; Armstrong, L.L.; Lee, J.Y.; Sisk, R.; Karaderi, T.; Barber, T.M.; McCarthy, M.I.; Franks, S.; et al. Genome-wide association of polycystic ovary syndrome implicates alterations in gonadotropin secretion in European ancestry populations. Nat. Commun. 2015, 6, 7502. [CrossRef] [PubMed]

11. Day, F.; Karaderi, T.; Jones, M.R.; Meun, C.; He, C.; Drong, A.; Kraft, P.; Lin, N.; Huang, H.; Broer, L.; et al. Large-scale genome-wide meta-analysis of polycystic ovary syndrome suggests shared genetic architecture for different diagnosis criteria. PLoS Genet. 2018, 14, e1007813. [CrossRef] [PubMed]

12. Zhang, Y.; Ho, K.; Keaton, J.M.; Hartzel, D.N.; Day, F.; Justice, A.E.; Josyula, N.S.; Pendergrass, S.A.; Actkins, K.; Davis, L.K.; et al. A genome-wide association study of polycystic ovary syndrome identified from electronic health records. Am. J. Obstet. Gynecol. 2020, 223, 559.e1-559.e21. [CrossRef] 
13. Udler, M.S.; Kim, J.; von Grotthuss, M.; Bonas-Guarch, S.; Cole, J.B.; Chiou, J.; on Behalf of METASTROKE and the ISGC; Boehnke, M.; Laakso, M.; Atzmon, G.; et al. Type 2 diabetes genetic loci informed by multi-trait associations point to disease mechanisms and subtypes: A soft clustering analysis. PLoS Med. 2018, 15, e1002654. [CrossRef]

14. Yaghootkar, H.; Scott, R.A.; White, C.C.; Zhang, W.; Speliotes, E.; Munroe, P.B.; Ehret, G.B.; Bis, J.C.; Fox, C.S.; Walker, M.; et al. Genetic evidence for a normal-weight "metabolically obese" phenotype linking insulin resistance, hypertension, coronary artery disease, and type 2 diabetes. Diabetes 2014, 63, 4369-4377. [CrossRef] [PubMed]

15. Dimas, A.S.; Lagou, V.; Barker, A.; Knowles, J.W.; Magi, R.; Hivert, M.F.; Benazzo, A.; Rybin, D.; Jackson, A.U.; Stringham, H.M.; et al. Impact of type 2 diabetes susceptibility variants on quantitative glycemic traits reveals mechanistic heterogeneity. Diabetes 2014, 63, 2158-2171. [CrossRef]

16. Locke, A.E.; Kahali, B.; Berndt, S.I.; Justice, A.E.; Pers, T.H.; Day, F.R.; Powell, C.; Vedantam, S.; Buchkovich, M.L.; Yang, J.; et al. Genetic studies of body mass index yield new insights for obesity biology. Nature 2015, 518, 197-206. [CrossRef] [PubMed]

17. Shungin, D.; Winkler, T.W.; Croteau-Chonka, D.C.; Ferreira, T.; Locke, A.E.; Magi, R.; Strawbridge, R.J.; Pers, T.H.; Fischer, K.; Justice, A.E.; et al. New genetic loci link adipose and insulin biology to body fat distribution. Nature 2015, 518, 187-196. [CrossRef]

18. Sun, B.B.; Maranville, J.C.; Peters, J.E.; Stacey, D.; Staley, J.R.; Blackshaw, J.; Burgess, S.; Jiang, T.; Paige, E.; Surendran, P.; et al. Genomic atlas of the human plasma proteome. Nature 2018, 558, 73-79. [CrossRef]

19. Manning, A.K.; Hivert, M.F.; Scott, R.A.; Grimsby, J.L.; Bouatia-Naji, N.; Chen, H.; Rybin, D.; Liu, C.T.; Bielak, L.F.; Prokopenko, I.; et al. A genome-wide approach accounting for body mass index identifies genetic variants influencing fasting glycemic traits and insulin resistance. Nat. Genet. 2012, 44, 659-669. [CrossRef] [PubMed]

20. Dupuis, J.; Langenberg, C.; Prokopenko, I.; Saxena, R.; Soranzo, N.; Jackson, A.U.; Wheeler, E.; Glazer, N.L.; Bouatia-Naji, N.; Gloyn, A.L.; et al. New genetic loci implicated in fasting glucose homeostasis and their impact on type 2 diabetes risk. Nat. Genet. 2010, 42, 105-116. [CrossRef]

21. Willer, C.J.; Schmidt, E.M.; Sengupta, S.; Peloso, G.M.; Gustafsson, S.; Kanoni, S.; Ganna, A.; Chen, J.; Buchkovich, M.L.; Mora, S.; et al. Discovery and refinement of loci associated with lipid levels. Nat. Genet. 2013, 45, 1274-1283. [CrossRef]

22. Xue, A.; Wu, Y.; Zhu, Z.; Zhang, F.; Kemper, K.E.; Zheng, Z.; Yengo, L.; Lloyd-Jones, L.R.; Sidorenko, J.; Wu, Y.; et al. Genome-wide association analyses identify 143 risk variants and putative regulatory mechanisms for type 2 diabetes. Nat. Commun. 2018, 9 , 2941. [CrossRef] [PubMed]

23. Van der Harst, P.; Verweij, N. Identification of 64 novel genetic loci provides an expanded view on the genetic architecture of coronary artery disease. Circ. Res. 2018, 122, 433-443. [CrossRef]

24. Michailidou, K.; Lindstrom, S.; Dennis, J.; Beesley, J.; Hui, S.; Kar, S.; Lemacon, A.; Soucy, P.; Glubb, D.; Rostamianfar, A.; et al. Association analysis identifies 65 new breast cancer risk loci. Nature 2017, 551, 92-94. [CrossRef]

25. Hemani, G.; Zheng, J.; Elsworth, B.; Wade, K.H.; Haberland, V.; Baird, D.; Laurin, C.; Burgess, S.; Bowden, J.; Langdon, R.; et al. The MR-base platform supports systematic causal inference across the human phenome. Elife 2018, 7, e34408. [CrossRef] [PubMed]

26. Zhang, Y.; Poler, S.M.; Li, J.; Abedi, V.; Pendergrass, S.A.; Williams, M.S.; Lee, M.T.M. Dissecting genetic factors affecting phenylephrine infusion rates during anesthesia: A genome-wide association study employing EHR data. BMC Med. 2019, 17, 168. [CrossRef] [PubMed]

27. Malika Charrad, N.G.; Boiteau, V.; Niknafs, A. NbClust: An R package for determining the relevant number of clusters in a data set. J. Stat. Softw. 2014, 61, 20875. [CrossRef]

28. Lotta, L.A.; Gulati, P.; Day, F.R.; Payne, F.; Ongen, H.; van de Bunt, M.; Gaulton, K.J.; Eicher, J.D.; Sharp, S.J.; Luan, J.; et al. Integrative genomic analysis implicates limited peripheral adipose storage capacity in the pathogenesis of human insulin resistance. Nat. Genet. 2017, 49, 17-26. [CrossRef]

29. Wang, Q.; Holmes, M.V.; Davey Smith, G.; Ala-Korpela, M. Genetic support for a causal role of insulin resistance on circulating branched-chain amino acids and inflammation. Diabetes Care 2017, 40, 1779-1786. [CrossRef]

30. Willer, C.J.; Li, Y.; Abecasis, G.R. METAL: Fast and efficient meta-analysis of genomewide association scans. Bioinformatics 2010, 26, 2190-2191. [CrossRef]

31. Li, L.; Cheng, W.Y.; Glicksberg, B.S.; Gottesman, O.; Tamler, R.; Chen, R.; Bottinger, E.P.; Dudley, J.T. Identification of type 2 diabetes subgroups through topological analysis of patient similarity. Sci. Transl. Med. 2015, 7, 311ra174. [CrossRef]

32. Ahlqvist, E.; Storm, P.; Karajamaki, A.; Martinell, M.; Dorkhan, M.; Carlsson, A.; Vikman, P.; Prasad, R.B.; Aly, D.M.; Almgren, P.; et al. Novel subgroups of adult-onset diabetes and their association with outcomes: A data-driven cluster analysis of six variables. Lancet Diabetes Endocrinol. 2018, 6, 361-369. [CrossRef]

33. Zhao, Y.; Xu, Y.; Wang, X.; Xu, L.; Chen, J.; Gao, C.; Wu, C.; Pan, D.; Zhang, Q.; Zhou, J.; et al. Body mass index and polycystic ovary syndrome: A 2-sample bidirectional mendelian randomization study. J. Clin. Endocrinol. Metab. 2020, 105, dgaa125. [CrossRef] [PubMed]

34. Brower, M.A.; Hai, Y.; Jones, M.R.; Guo, X.; Chen, Y.I.; Rotter, J.I.; Krauss, R.M.; Legro, R.S.; Azziz, R.; Goodarzi, M.O. Bidirectional Mendelian randomization to explore the causal relationships between body mass index and polycystic ovary syndrome. Hum. Reprod. 2019, 34, 127-136. [CrossRef]

35. Velazquez, E.M.; Mendoza, S.; Hamer, T.; Sosa, F.; Glueck, C.J. Metformin therapy in polycystic ovary syndrome reduces hyperinsulinemia, insulin resistance, hyperandrogenemia, and systolic blood pressure, while facilitating normal menses and pregnancy. Metabolism 1994, 43, 647-654. [CrossRef] 
36. Facchinetti, F.; Unfer, V.; Dewailly, D.; Kamenov, Z.A.; Diamanti-Kandarakis, E.; Lagana, A.S.; Nestler, J.E.; Soulage, C.O.; Group of 'Inositol in, PCOS and Reproduction'. Inositols in polycystic ovary syndrome: An overview on the advances. Trends Endocrinol. Metab. 2020, 31, 435-447. [CrossRef]

37. Lagana, A.S.; Rossetti, P.; Sapia, F.; Chiofalo, B.; Buscema, M.; Valenti, G.; Rapisarda, A.M.C.; Vitale, S.G. Evidence-based and patient-oriented inositol treatment in polycystic ovary syndrome: Changing the perspective of the disease. Int. J. Endocrinol. Metab. 2017, 15, e43695. [CrossRef] [PubMed]

38. Ehrmann, D.A.; Barnes, R.B.; Rosenfield, R.L.; Cavaghan, M.K.; Imperial, J. Prevalence of impaired glucose tolerance and diabetes in women with polycystic ovary syndrome. Diabetes Care 1999, 22, 141-146. [CrossRef] [PubMed]

39. Legro, R.S.; Kunselman, A.R.; Dodson, W.C.; Dunaif, A. Prevalence and predictors of risk for type 2 diabetes mellitus and impaired glucose tolerance in polycystic ovary syndrome: A prospective, controlled study in 254 affected women. J. Clin. Endocrinol. Metab. 1999, 84, 165-169. [CrossRef] [PubMed]

40. Carvalho, M.J.; Subtil, S.; Rodrigues, A.; Oliveira, J.; Figueiredo-Dias, M. Controversial association between polycystic ovary syndrome and breast cancer. Eur. J. Obstet. Gynecol. Reprod. Biol. 2019, 243, 125-132. [CrossRef]

41. Harris, H.R.; Terry, K.L. Polycystic ovary syndrome and risk of endometrial, ovarian, and breast cancer: A systematic review. Fertil. Res. Pract. 2016, 2, 14. [CrossRef] [PubMed]

42. Mihm, M.; Gangooly, S.; Muttukrishna, S. The normal menstrual cycle in women. Anim. Reprod. Sci. 2011, 124, 229-236. [CrossRef] [PubMed] 